\title{
Review
}

\section{Marcel Broodthaers ein Maler? Über die Sonderstellung eines Mediums unter post-medialen Bedingungen}

Isabelle Graw: Die Liebe zur Malerei: Genealogie einer Sonderstellung. Zürich: Diaphanes, 2017. 399 S.

Rezensiert von Malte Fabian Rauch: Humboldt Universität zu Berlin, Institut für Philosophie, Unter den Linden 6, D-10099 Berlin, Deutschland, E-Mail: raucm763@newschool.edu

https://doi.org/10.1515/arcadia-2018-0015

\section{Malerei und Post-Medialität}

Isabelle Graw hat ein Buch über die Malerei geschrieben, über ihre Sonderstellung. Es ist ein ambitioniertes Buch, ein persönliches. Auch ein streitbares. Denn es gilt wohl noch immer, dass wer im Kontext avancierter Kunsttheorie von Malerei sprechen will, Rechenschaft ablegen muss über Legitimität und Aktualität seines Themas. Wie sonst nur der Roman, ist die Malerei eine künstlerische Praxis, deren Tod im 20. Jahrhundert Konjunktur hatte. Erklärt wurde er erstmals aus einer der Malerei immanenten Position, in ihren von den Avant-Garden inszenierten Endspielen in der ersten Hälfte des Jahrhunderts. Dann, seit den 1960er Jahren, von Seiten von Kritiker/innen und Künstler/innen aus ganz unterschiedlichen Lagern. An ihr festzuhalten, so erzählte man sich seit dieser Zeit jedenfalls vernehmlich, mit bornierter Regelmäßigkeit, alle Jahre wieder und in den nämlichen Variationen, sei künstlerisch nunmehr uninteressant, politisch regressiv. Mal galt die Malerei als eine Praxis, deren Innovationsmöglichkeiten erschöpft sind, die sich in Wiederholungen und Epigonentum verliert und offensichtlich zur Ware verkommen ist, mal als einem problematischen Begriff individuellen Ausdrucks verhaftet, dann wieder als unheilbar illusionistisch. Schließlich als überholte, weil vom linguistischen Register ausgeschlossene Kunstform. Was dabei entschieden schien, war ihre Obsoleszenz, die radikale Hinfälligkeit ihrer historischen Existenzberechtigung.

Prekär scheint ihre Stellung infolge der Transformation, welche die Kunst in den 1960er Jahren durchlaufen hat. Denn ignorierte man, wie bis dahin üblich, die 
Konsequenzen, die vor allem Dada für den Kunstbegriff hätte haben müssen, dann mochte es scheinen, als seien die bisherigen Innovationen und Negationen moderner Kunst stets im Rahmen bestimmter Medien verblieben. Also konnte ein auf Medienspezifik insistierender Formalismus samt dem dazugehörigen Verständnis künstlerischer Autonomie als dominanter Theoriediskurs auftreten; er war, zumal in den USA, ohne Frage dessen „master narrative“. ${ }^{1}$ Auch, welche Bedeutung dies für die Malerei hatte, lässt sich am deutlichsten in den USA, an der New Yorker Szene nachvollziehen. Anfang der 1960er Jahre galt dem seinerzeit hegemonialen Formalismus von Clement Greenberg und seinem Adepten Michael Fried die Malerei noch unbestritten als die richtungsweisende Kunstform. Konnte doch vor allem Greenbergs Theorie der Medienspezifik überhaupt nur in einer selektiven Lesart der Malerei von Édouard Manet bis zum Abstrakten Expressionismus plausibel gemacht werden, und bezog ihre Legitimität allein aus jener. Nur für die Malerei konnte nämlich ein Narrativ konstruiert werden, in dem ihre Entwicklung beschreibbar wird als Flucht aus der Repräsentation in die Reflexion auf das Trägermedium, eine Entwicklung im Zuge derer das Wesen des Mediums definiert worden sei. Allein von der Malerei hatte Greenberg seine gesamte Theorie der Medienspezifik abgezogen und als Theorie moderner Kunst schlechthin präsentiert.

Spätestens seit Mitte der 1960er war gleichwohl absehbar, dass eine neue Entwicklung auf dem Weg war, eine Entwicklung zu intermedialen Praktiken, zu Installationen, Performances, Video und konzeptuellen Arbeiten. Zu Kunst, welche die Moderne historisch und den Formalismus akademisch werden ließ. Man kann diese Geschichte, die Geschichte der Kunst der 1960er Jahre, die eine Geschichte des Spiels mit den Grenzen der Medien ist, als eine unübersichtliche erzählen, oder man kann sie so erzählen, dass sie recht direkt von Frank Stellas Black Paintings von 1959 über die Diskussion um den Minimalismus zum Anspruch einer Dematerialisierung des Objekts in der Konzeptkunst führt. ${ }^{2}$ Gleichviel. Wichtig allein, was an ihrem Ende stand. Und dabei scheint der Begriff der post-medium condition, den Rosalind Krauss in ihren Walter-Neurath-Vorlesungen zur Beschreibung der Situation prägte, bekanntlich ohne sie zu begrüßen, die einzige deskriptive Kategorie zu sein, auf die sich theoretisch noch geeinigt werden kann. ${ }^{3}$ Denn nun galt die Malerei den meisten als passé und für die

1 Rosalind E. Krauss: Perpetual Inventory. Cambridge, MA: MIT Press, 2013. xiii.

2 Vgl. exemplarisch für die mittlerweile recht etablierte Genealogie Stella/Minimalismus/Konzeptkunst: Thierry De Duve: Kant after Duchamp. Cambridge, MA: MIT Press, 1996. Kap. 4.

3 Rosalind E. Krauss: A Voyage on the North Sea: Art in the Age of the Post-Medium Condition. London: Thames \& Hudson, 2000. Eine wirkliche Auseinandersetzung mit Krauss' Modell bleibt jedoch aus: In diesem wird nicht, wie von Graw an zwei Stellen behauptet, von gegebenen bzw. feststehenden Konventionen ausgegangen (143 und 379, Fn. 1). Auch Krauss' neuere Arbeiten zum 
Entwicklung einer Kunst, die keine moderne mehr sein wollte, als irrelevant. Die Sonderstellung war dahin. Ihr Posten ein verlorener. Denn was könnte sie, vormaliges Paradigma eines medienspezifischen Verständnisses von Kunst, in einer postmedialen Situation noch sein? Trauerarbeit, Meditationen über ihr Ende, vielleicht noch dies, schrieb Mitte der 1980er Jahre jemand, der es wissen musste. ${ }^{4}$ Und Robert Ryman war der berufene Grabwächter.

Der Status von Malerei hat sich seitdem verändert. Beginnend in den 1980er Jahren, im Zuge einer massiven Rückkehr figurativer Ansätze, und dann mit zunehmender Sichtbarkeit in den 1990er Jahren, hat sie in diversen Teilen der Kunstwelt an Terrain gewonnen. Um die Jahrhundertwende war schließlich unübersehbar, dass sich die Malerei institutionell konsolidiert hatte. ${ }^{5}$ Der Verdacht allerdings, der in den 1960er und 1970er Jahren formuliert wurde, Malerei sei heillos reifiziert, bar jeden Anspruchs jenseits der Wanddekoration, ist in manchen theoretischen Lagern nie fallen gelassen worden. Die skeptische Frage nach dem Status der Malerei unter post-medialen Bedingungen ist mithin geblieben, zumal als Ausgangspunkt für jede theoretisch gehaltvolle Auseinandersetzung mit ihr. Bei Isabelle Graw, die über einen meisterhaften Überblick sämtlicher in diesem Zusammenhang relevanter Entwicklungen verfügt, steht daher allein ein Begriff der Malerei „unter post-medialen Bedingungen“ zur Diskussion (10). Den Fragen und Problemen, die sich daran anschließen, begegnet sie zunächst mit einer Beobachtung und einer terminologischen Unterscheidung. „Theoretisch“, so Graw, mag die These einer Entgrenzung der Künste zwar zutreffend sein (33); aber dies steht im krassen Widerspruch dazu, welchen Status die Kunstform realiter noch immer einnimmt, zu den Preisen, die bei Auktionen für sie gezahlt, zu der Anzahl an Ausstellungen, die ihr gewidmet werden. Darum wird von ihr

Thema werden nicht diskutiert. Vgl. dazu „Reinventing the Medium.“ Critical Inquiry 25.2 (1999): 289-305; „Two Moments from the Post-Medium-Condition.“ October 116 (2006): 55-62; Under Blue Cup. Cambridge, MA: MIT Press, 2011.

4 „Painting might not be dead. Its vitality will only be tested once we are cured of our mania and our melancholy, and we believe again in our ability to act in history: accepting our project of working through the end again, [...] and settling our historical task: the difficult task of mourning.“ Yves-Alain Bois: „Painting: The Task of Mourning.“ 1986. Painting as Model. Cambridge, MA: MIT Press, 1990. 229-244; 243.

5 Zwei Beispiele aus dem Jahr 2002: Der erste Vitamin P1 Katalog bot einen Überblick über die neue internationale Konstellation der Malerei, und eine Ausstellung im Centre Georges Pompidou, die danach in der Kunsthalle Wien und der Kunsthalle Schirn in Frankfurt zu sehen war, widmete sich mit hohem kuratorischen Aufwand der Neubewertung figurativer Malerei. Vgl. Válerie Breuvart, Hg.: Vitamin P1: New Perspectives in Painting. London und New York: Phaidon, 2002; Centre Georges Pompidou et al., Hg.: „Lieber Maler, male mir ...“: Radikaler Realismus nach Picabia. Ausstellungskatalog. Paris: Centre Georges Pompidou et al., 2002. 
„gleichsam kontraintuitiv“ der „soziologische[] Befund einer Sonderstellung der Malerei unter post-medialen Bedingungen“ erhoben (10).

Das Buch beginnt mit einer Einleitung, in welcher der ambitionierte theoretische Rahmen exponiert wird, und gliedert sich dann in sechs Kapitel, die jeweils ein Thema unter verschiedenen Aspekten behandeln. Einige der argumentativen Linien des Buches sind dem Leser der Aufsätze, die Graw in den letzten Jahren zur Malerei veröffentlicht hat, bekannt; sie finden hier allerdings in einer neuen Konstellation zusammen, womit das Buch so etwas wie eine Summe der vorherigen Studien darstellt. Graw formuliert ihre methodische Fragestellung mit einer seltenen Präzision und konfrontiert die Probleme, die sich aus ihr ergeben, mit einer ebenso raren Direktheit. Aus diesem Grund soll hier keine konventionelle Rezension mit summarischem und distanziertem Gestus vorgelegt werden. Vielmehr wird Graws theoretischer Zugang als Punkt genommen, in dem sich diverse generelle Fragen und Probleme der zeitgenössischen Theoriebildung konzentrieren und als solche exemplarisch diskutierbar werden (Abschnitt 2). Im Anschluss daran wird - gleichsam synoptisch - auf die einzelnen Kapitel des Buches einzugehen sein, wobei die Analyse des theoretischen Rahmens in den Hintergrund treten kann (Abschnitt 3).

\section{Malerei als Formation, Malerei als System von Zeichen?}

Mit der Exposition ihres theoretischen Rahmens beginnt Graw eine Erklärung für den soziologischen Befund einer fortbestehenden Sonderstellung der Malerei unter post-medialen Bedingungen zu formulieren. Dabei steht das Modell unter doppeltem Zugzwang. Einerseits gilt es, die diversen, wohl bekannten und an dieser Stelle nicht zu wiederholenden Aporien eines medienspezifischen Formalismus zu vermeiden. ${ }^{6}$ Andererseits muss, wer an einem Begriff von Malerei als distinkter Praxis auch unter post-medialen Bedingungen noch festhalten will, Kriterien angeben können, die sich nicht in radikalem Nominalismus oder Transkategorialität auflösen. Graws theoretische Strategie besteht aus drei Schritten. In einem ersten wird der Begriff der Malerei historisiert, in einem zweiten semiotisch reformuliert, in einem dritten schließlich durch eine werttheoretische Perspektive komplettiert.

Für den ersten Schritt, die Historisierung der Malerei, greift Graw auf Michel Foucaults Begriff der Formation zurück. Also wird Malerei:

6 Vgl. Thierry De Duve: Clément Greenberg entre les lignes. Paris: Dis Voir, 1995. 


\begin{abstract}
als eine „Formation“ bestimmt, was ihrer Spezifik bei gleichzeitiger Entspezifizierung Rechnung trägt. Formationen sind Foucault zufolge historisch gewachsene und veränderliche Gebilde, die zugleich bestimmten „Formationsregeln“ gehorchen. Der Fokus meiner Untersuchung liegt dementsprechend auf der Genese der Formation „Malerei“, auf den historischen Veränderungen, die sie durchlief, wie auch auf dem, was sie durchgängig auszeichnet. Es ist die Stärke des Formationsbegriffes, dass er Veränderungen, Öffnungen und Grenzverschiebungen ebenso zu denken erlaubt wie das kontinuierlich Bestehende. (14)
\end{abstract}

Statt sie als etwas Gegebenes, als eine substanzielle, der Zeit entzogene Entität zu begreifen, wird Malerei mithin als ein Ensemble von Praktiken, Institutionen und Regeln begriffen, die vor allem auf die Genese des Tafelbildes zwischen dem 15. und 18. Jahrhundert zurückgeführt wird (344). Damit definiert die „Genealogie“ dieser Formation, ihre historischen Entstehungsbedingungen und ihr Sonderstatus Graws Programm $(16,19)$. Doch die Autorin möchte diese genealogische Rekonstruktion nicht als skeptische Ideologiekritik verstanden wissen. Neben der gebotenen Historisierung und Distanzierung des Gegenstandes wird vielmehr davon ausgegangen, dass dieser auch über ein gewisses Potenzial verfüge, die Betrachterin zu affizieren, eine Fähigkeit, auf die sich bewusst eingelassen werden soll (1617).

Was bei diesem methodischen Bezug auf Foucault allerdings ungeklärt bleibt, ist Graws lakonische Erklärung, im Gegensatz zu dessen Modell werde bei ihr die Kategorie des Subjekts eine zentrale Position einnehmen (15). Auf Graws Studie trifft dies freilich zu, ohne Frage entsteht bei ihr „die Formation ,Malerei“ nicht quasi-automatisch, wie bei Foucault, sondern aufgrund bestimmter Praktiken, die ihrerseits bestimmte Produkte hervorbringen und einzelnen Akteur/innen zugeschrieben werden“ (15). Allein, wie soll dieser Anspruch auf eine Historisierung im Sinne Foucaults und das Insistieren auf einem nichtlinearen, diskontinuierlichen Geschichtsmodell mit der Konzeption des Subjekts als Akteur, der Formationen erzeuge, zusammengebracht werden? Nicht die Frage nach der Legitimität der Subjektkategorie in der Kunstgeschichte als solcher soll hier entschieden werden, wohl aber jene der Systematik von Foucaults Methode. Denn dessen innovative Wendung an der schon seinerzeit konventionellen Kritik eines linearen, letztlich also idealistischen Geschichtskonzepts hatte ja gerade in dem Argument bestanden, dass der Begriff des konstitutiven Subjekts und jener der kontinuierlichen Geschichte „unerläßliche Korrelat[e]“ sind, sich also wechselseitig implizieren. ${ }^{7}$ Und gerade in dieser Reziprozität sollten sie

7 „Die kontinuierliche Geschichte ist das unerläßliche Korrelat für die Stifterfunktion des Subjekts [...]. Aus der historischen Analyse den Diskurs des Kontinuierlichen machen und aus dem menschlichen Bewußtsein das ursprüngliche Subjekt allen Werdens und jeder Anwendung machen, das sind die beiden Gesichter ein und desselben Denksystems.“ Michel Foucault: 
durch Archäologie und Genealogie aufgelöst, zersetzt werden. Was Graw an dieser Stelle schuldig bleibt, ist eine Erklärung, wie der so formulierte Eingriff in Foucaults Ansatz zu verstehen ist. Wer das Subjekt als konstitutives Element wieder zulässt, wird sich auf Foucaults Formulierung eines diskontinuierlichen Geschichtsbegriffs kaum berufen können. Ihm war das Subjekt das in der Geschichte Konstituierte, nicht das sie konstituierende, das Innen das „Werk des Außen". ${ }^{8}$

In einem zweiten Schritt nimmt Graw eine semiotische Perspektive auf die Malerei ein, im Kontext derer sie auch die übrigen für ihre Analyse zentralen Begriffe einführt. Ausgehend von einer besonderen „Affektwirkung“, welche der Malerei, wie Graw unschwer belegen kann, historisch zugeschrieben wurde, fragt sie nach den Bedingungen, die dieses Potenzial erklären mögen (21). Dazu wird von ihr ein semiotischer Zugang formuliert, in dem Malerei als ein „Zeichensystem“ konzipiert wird, denn zwischen „Zeichen und ihrer Affektwirkung“ bestehe eine „tiefe Verbindung“ (21). Was die Besonderheit des malerischen Zeichens ausmacht, ist laut Graw, dass „[s]eine physikalische Form, seine Materialität und Körperlichkeit, stets im Vordergrund steht“ (21). Diese begriffliche Konstellation führt $\mathrm{zu}$ dem Begriff, der in Graws Buch zum zentralen Paradigma wird, zur Klammer, welche Geschichte und Gegenwart der Malerei zusammenhält. Denn gerade an der dem malerischen Zeichen eigenen Akzentuierung der Materialität entzünde sich das, was Graw „vitalistische Phantasien“ nennt. Darunter wird die Suggestion vitaler Eigenschaften wie „Subjektivität, Lebenskraft und Beseeltheit" verstanden, wobei deren Evokation in der Malerei der Projektion menschlicher Attribute wie „Souveränität, Eigenwilligkeit oder Selbständigkeit auf leblose Materie“ gleichkomme (23). Der Begriff der Vitalität wird bei Graw schillernd, was einerseits seine Attraktivität ausmacht, andererseits zu methodischen Problemen führt, von denen noch zu sprechen sein wird. In einer ersten Annäherung lassen sich drei zentrale Bedeutungen ausmachen, die bei Graw allerdings zum Teil ineinander übergehen. Erstens meint Lebendigkeit Spielarten einer Vorstellung, gemäß derer die Person der Künstlerin auf eine gewisse Weise in der Malerei präsent sei (23). Zweitens meint der Begriff den Topos einer Autonomie des Bildes, welcher sich in der Kunstkritik ebenso findet wie in der Bildwissenschaft.

Archäologie des Wissens. Die Hauptwerke. Frankfurt a.M.: Suhrkamp, 2008. 471-699; 486-487. Vgl. grundsätzlich zu diesem Themenfeld bei Foucault: Reiner Schürmann: „On Constituting Oneself an Anarchistic Subject." 1986. Tomorrow the Manifold: Essays on Foucault, Anarchy, and the Singularization to Come. Hgg. Malte Fabian Rauch und Nicolas Schneider. Berlin und Zürich: Diaphanes. Im Druck.

8 Gilles Deleuze: Foucault. 1986. Frankfurt a.M.: Suhrkamp, 1992. 135. 
Drittens meint Lebendigkeit, und das im merklichen Gegensatz zu den beiden vorherigen Bedeutungen, eine Art Meta-Kategorie, die zwischen Produktionsästhetik und Beschreibung der historischen Dynamik von Malerei changiert. Graw bezeichnet diese Variante als „Lebendigkeit zweiter Ordnung [...] eine Art Lebendigkeit, die den Aspekt ihrer Herstellung betont und sich als Effekt eines malerischen Verfahrens präsentiert“ (25, Kursive im Original). Dieser Aspekt soll vor allem der Tatsache Rechnung tragen, dass Lebendigkeit als explizites Kriterium in der Moderne zwar an Bedeutung verloren hat, als implizites gleichwohl weiterhin von großer Relevanz sei, und zwar vor allem immer dann, wenn die Malerei durch nichtmalerische Strategien belebt wurde. Was soll das heißen? In ihrer exponiertesten Form ist Graws These, und es ist die entscheidende des Buches, dass wann „immer sich die Malerei hin zu anderen Medien [...] öffnete, heterogen wurde und sich entspezifizierte, vermochte sie im gleichen Zuge erst recht ihre Sonderstellung auszubauen“ (49). Die spekulative Wette des Buches lautet also, dass noch jede Negation der Malerei in deren Revitalisierung terminierte. Wie sogleich zu zeigen sein wird, ist es diese heterogene, ja konfligierende Semantik des Lebendigkeitsbegriffs, welche dem Modell in der Entfaltung des Arguments Probleme bereitet.

Im Übrigen wäre mit Blick auf die These, dass der Topos der Lebendigkeit besonders für die Malerei charakteristisch ist, abschließend noch zu fragen, ob er in diesem Zusammenhang nicht als Teil eines generellen Diskurses in der neuzeitlichen Ästhetik verstanden werden sollte, womit er als Distinktionsmerkmal gerade ungeeignet wäre. Apodiktisch spricht Kant, um nur ein Beispiel zu nennen, ja etwa davon, dass nicht die Malerei, sondern das Schöne insgesamt „directe ein Gefühl der Beförderung des Lebens bei sich führt“. 9

Theoretisch ist das Modell nicht ohne Charme. Das semiotische Verständnis der Malerei, welches ihre Konventionen als ein Ensemble analysierbarer Zeichen fasst, scheint sich zunächst problemlos in das historistische Geschichtsmodell zu fügen, welches die Bedeutung von Institutionen, den ihnen immanenten Regeln und Praktiken akzentuiert. So wird eine Perspektive eröffnet, die es erlaubt, an einer, wie Graw treffend formuliert, nicht-essenzialistischen „Rest-Spezifik“ von Malerei auch unter post-medialen Bedingungen festzuhalten (14). Zwei kritische Fragen müssen gleichwohl an diese Theorie gestellt werden; sie betreffen das Verhältnis des semiotischen Modells zum formalistischen Begriff der Medienspezifik und die theoretische Architektur des Zeichenbegriffs, dessen Indexikalität.

9 Immanuel Kant: Kritik der Urteilskraft. Hg. Heiner F. Klemme. Hamburg: Felix Meiner, 2009. 106. Als Überblick zu diesem Diskurs vgl. etwa Armen Avanessian, Winfried Menninghaus und Jan Völker, Hgg.: Vita aesthetica: Szenarien ästhetischer Lebendigkeit. Zürich und Berlin: Diaphanes, 2009. 
Erstens ist zu fragen, ob der formalistische Begriff der Medienspezifik, gegen den dieses Modell antritt, hier nicht ein Nachleben führt, welches das semiotische Vokabular nur spärlich kaschieren kann. Denn offensichtlich tritt der Begriff des Zeichens systematisch exakt an die Stelle dessen, was bei Greenberg die „irreducible essence of pictorial art“ hieß und bekanntlich in der „flatness and the delimitation of flatness“ bestehen sollte (vgl. 56). ${ }^{10}$ Das Wesen der Malerei sollte für Greenberg mithin in der Thematisierung ihres Trägermediums bestehen, welches brav-kantianisch als Bedingung der Möglichkeit der Praxis konzipiert war. Nun zieht Graw mit ihrer Strategie von Historismus und Semiotik alle theoretischen Register, um auch nur den Anschein eines essenzialistischen Vokabulars zu vermeiden. Allein: Wer „Besonderheit des malerischen Zeichens“ (21) sagt, wer „mediale und materiale Eigenheit von Gemälden“ (17, vgl. 43) sagt, sagt nichts anderes als Spezifik des Mediums. Und bestimmt wird die Spezifik der Malerei, die bei Graw als Spezifik des malerischen Zeichens figuriert, zunächst durch zwei Merkmale. Erstens als die bereits erwähnte Betonung der „Materialität und Körperlichkeit“ (21), später dann aber auch als eine „spezifische[] Form der Indexikalität“ (343), wobei nie recht klar wird, ob das zweite Merkmal sub specie des ersten oder unabhängig von ihm ist.

Worin, so muss jedenfalls gefragt werden, besteht denn der Unterschied zwischen einer Theorie, welche die Spezifik anhand des Wesensbegriffs denkt und auf die Akzentuierung der Flächigkeit des Tafelbildes reduziert, und einer vermeintlich alternativen, bei der die Spezifik über den Zeichenbegriff, dessen Thematisierung der Materialität und Indexikalität konstruiert wird? Steht diese Konstruktion nicht dem historistischen Geschichtsmodell entgegen, für das Graw optiert, da letztlich ungeklärt bleibt, was an der Formation Malerei historistisch Gewordenes, was Vergehendes ist, wenn der Begriff des malerischen Zeichens, auf den diese gründet, als Invariante formuliert wird? Und lassen sich schließlich nicht auch diverse Werke zitieren, bei denen die Betonung von Lebendigkeit und Materialität kritisiert, ja parodiert wird? Was den letzten Punkt betrifft, ist sich Graw aller totzitierten Gegenbeispiele wie László Moholy-Nagys Telefonbildern aus den 1920er Jahren freilich bewusst. Mehr noch, Graw sucht solche immanen-

10 Clement Greenberg: „After Abstract Expressionism.“ 1962. The Collected Essays and Criticism. Hg. John O’Brian. Bd. IV. Chicago: U of Chicago P, 1996. 121-134. Im Übrigen hatte selbst Michael Fried in seinem bereits aus der panischen Defensive formulierten Versuch einer Restauration der Medienspezifik seinen Lehrer Greenberg kritisiert und auf eine Historisierung und Entsubstanzialisierung des Wesensbegriffs gedrängt: „[E]ssence - i.e., that which compels conviction - is largely determined by, and therefore changes continually in response to, the vital work of the recent past. The essence of painting is not something irreducible.“ Michael Fried: „Art and Objecthood.“1967. Art and Objecthood. Chicago: Chicago UP, 1998. 148-172, hier 168-169, Fn. 6. 
ten Kritiken der Malerei sogar für ihre Theorie der vitalistischen Phantasien zu rekrutieren, und zwar mit dem Argument, dass es bei diesen Arbeiten die erwähnte „Lebendigkeit zweiter Ordnung“ sei, die zur Geltung komme. Dabei zeigt sich jedoch die ganze Problematik eines so gefassten Lebendigkeitsbegriffs, denn es ist keineswegs nachvollziehbar, was diese Form von Lebendigkeit mit dem skizzierten Begriff des malerischen Zeichens noch zu tun haben soll.

So betrifft auch die zweite Frage die Struktur des Zeichenbegriffs, der hier vorausgesetzt wird, genauer: den Status der Indexikalität. Der von Graw formulierte Zugang weicht ab von der in der Kunstgeschichte noch immer dominanten, von Ferdinand de Saussure und seiner Rezeption im Strukturalismus und Poststrukturalismus ausgehenden Semiotik. ${ }^{11}$ In dieser Theorietradition, deren wichtigsten Vertreter in der Kunstgeschichte Hubert Damisch und die Strukturalisten im Umfeld des Journals October sind, ist das Verhältnis von Signifikant und Signifikat bekanntlich als arbiträres konzipiert. Die Bedeutung eines Zeichens wird nicht durch dessen Referenz oder die dem Signifikanten inhärenten Qualitäten bestimmt, sondern allein relational, durch die Position des Zeichens in einem differenziellen, in Oppositionen organisierten System.

Mit dem Begriff des Indexes und dem beiläufigen Verweis auf die Konzepte Ikone und Symbol optiert Graw für die theoretische Alternative zu diesem Modell, die Semiotik von Charles Sanders Peirce. ${ }^{12}$ Peirce' Zeichentheorie war lange Zeit in der Fotografietheorie dominant, wird in der Kunstgeschichte aber noch immer nur zögerlich als Alternative zu de Saussure wahrgenommen. Es sei dahingestellt, ob die dabei kanonisierte Lesart von Peirce' Theorie jene adäquat wiedergibt, ${ }^{13}$ entscheidend ist an dieser Stelle lediglich, dass ihr zufolge Indexikalität einen direkten physischen Bezug zwischen Zeichen und Bezeichnetem impliziert (wobei der strittige Punkt die Frage betrifft, ob dieser Bezug kausal verstanden werden muss oder nicht). In Analogie dazu soll der „malerische Index“ bei Graw nun nicht auf ein Objekt, sondern auf das Künstlersubjekt verweisen (110). Dabei soll jedoch nicht an dem vollen Index-Begriff festgehalten werden, vielmehr soll die Indexikalität des malerischen Zeichens als Suggestion verstanden werden, eine Suggestion, die die „Quasi-Anwesenheit des Autors als Effekt“ fabriziert (52). Ein

11 Vgl. zum Hintergrund der Rezeption Yves-Alain Bois: „Formalism and Structuralism.“ Art Since 1900: Modernism - Antimodernism - Postmodernism. Hgg. Benjamin H. D. Buchloh et al. 2. Aufl. New York: Thames \& Hudson, 2011. 32-39.

12 Zur dieser methodischen Opposition siehe Margaret Iverson: „Saussure versus Peirce: Models for a Semiotics of Visual Art.“ The New Art History. Hgg. A. L. Rees und F. Birgello. Atlantic Highlands, NJ: Humanities Press International, 1988. 82-94.

13 Vgl. James Elkins: „What Does Peirce’s Sign System Have to Say to Art History?“ Culture, Theory, and Critique 44:1 (2003): 5-22. 
Effekt mithin, der keinen physischen Bezug impliziert. ${ }^{14}$ Doch wenn der Indexbegriff abgelöst wird von der Idee einer solchen Relationalität, dann droht das Verhältnis zwischen Künstler/in und Werk theoretisch ebenso undurchsichtig zu werden wie der indexikalische Zeichenbegriff konturlos. So verstärkt sich der Eindruck weiter, dass dessen Funktion in der Substitution des medienspezifischen Wesensbegriffs besteht, ohne allerdings die sich daran anschließenden Probleme lösen zu können.

Ergänzt wird die so entworfene Konzeption schließlich durch eine von Karl Marx ausgehende Werttheorie. Bekanntlich hat Marx den Wert einer Ware durch das Quantum an abstrakter, das heißt gesellschaftlich notwendiger Arbeitszeit bestimmt, das zu ihrer Produktion erforderlich ist, um dann in der wohl berühmtesten Passage des Kapitals zu monieren, dass eben dieser gesellschaftliche Zusammenhang durch den „mystischen[n] Charakter“ der Waren, den Schein ihres Ansichseins verdeckt werde. ${ }^{15}$ Die interessante Wendung, die Graw ihrer häretischen Marx-Lektüre gibt, besteht nun in der Behauptung, dass in der Malerei der Arbeitsprozess gerade nicht verdeckt, sondern als solcher thematisiert werde, als Arbeit, die im Produkt unmittelbar enthalten sei. Und zwar vor allem durch die Suggestion vorkapitalistischer Arbeitsbedingungen, der Phantasie einer selbstbestimmten, körperlich-materiellen Arbeit am Bild. Thesen, die abgesehen von der werttheoretischen Dimension keineswegs aktuell sind, sondern auf Meyer Schapiros einflussreiche Interpretation der abstrakten Malerei als Antithese der industrialisierten Arbeit zurückgehen. ${ }^{16}$ Überzeugen kann die Verbindung dieser Thesen mit der Werttheorie aber vor allem deshalb, weil Graw derartige Vorstellungen damit weder affirmiert noch zur Illusion erklärt, sondern ihren Lebendigkeitsbegriff so um eine materialistische Komponente ergänzt, welche den kommerziellen Erfolg der Malerei in den Termini ihres theoretischen Rahmen fassbar macht.

14 Vgl. die konjunktivischen Formulierungen im Buch $(171,343)$ und die konzise Darstellung in Isabel Graw: „The Economy of Painting: Notes on the Vitality of a Success Medium and the Value of Liveliness." Painting 2.0: Expression in the Information Age. Ausstellungskatalog. Hgg. Manuela Ammer, Achim Hochdörfer und David Joselit. München et al.: Museum Brandhorst et al., 2015. 260-261.

15 Karl Marx: Das Kapital: Kritik der politischen Ökonomie. Bd. I. Marx-Engels-Werke. Bd. XXIII. Berlin: Dietz, 1962. 85.

16 Meyer Schapiro: „Recent Abstract Painting.“ 1957. Modern Art 19 \& 20th Centuries: Selected Papers. Bd. II. New York: George Braziller, 1982. 213-226, hier 217-218 und 222. In der Form recht fragwürdiger Aktualisierungen findet sich Shapiros Position noch heute wieder, so zuletzt bei Morgan Falconer: Painting beyond Pollock. New York: Phaidon, 2015. 11. 


\section{Von Spezifizierung und Entspezifizierung: Die Dialektik der Malerei}

In sechs Kapiteln elaboriert Graw diesen theoretischen Rahmen in einer thematisch je unterschiedlichen Perspektive. Das erste Kapitel beginnt mit einer Diskussion von Édouard Manet, dem Maler, der als Beginn der modernen Malerei kanonisiert wurde und in dieser Rolle den unumgänglichen Referenzpunkt für jede kunstgeschichtliche Auseinandersetzung mit dem Thema in den letzten Dekaden dargestellt hat. Mit deutlichem Bezug auf T. J. Clarks einflussreiche Interpretation, nach der sich Manets Werk nicht verstehen lasse, ohne dass die älteste Opposition in der Ästhetik, jene zwischen Form und Inhalt, aufgelöst und die Kategorien in ihrer Reziprozität behandelt werden, ist Graw bemüht, eine analoge Lesart in ihr Theoriemodell zu integrieren (61). ${ }^{17}$ Manets Betonung der Materialität der Malerei deutet sie, wie die formalistische Interpretation von Greenberg und seiner Schule, ${ }^{18}$ als Betonung der Spezifik des Mediums (65), um in Abgrenzung zu dieser die „massive Aufladung mit gesellschaftlichen Bedingungen“ dann als Verunreinigung eben dieser Spezifik zu deuten. Aus dem Widerspruch dieser beiden Tendenzen konstruiert sie die erwähnte Dialektik, nach der in der Moderne Entspezifizierung und Spezifizierung der Malerei in eins fallen. Das Kapitel schließt mit einem Gespräch zwischen Graw und Jutta Koether, dessen Gegenstand die Malerei von Joan Mitchell bildet.

Das zweite Kapitel, „Vom Deskilling zum Reskilling in subjekthaften Bildern“, beginnt mit der Diskussion einer weiteren kanonischen Figur der jüngeren Malereigeschichte: von Frank Stella und seinen Black Paintings aus den späten 1950er Jahren. Entgegen der zuletzt gängigen Auslegungen dieser Werkgruppe, die sie zum Endspiel und reductio ad absurdum des Formalismus erklärt, zu einer Form von Malerei, welche gen Objekthaftigkeit tendiere und als solche den Minimalismus antizipiere, ${ }^{19}$ liest Graw sie als Ausdehnung der räumlichen Geltungsmacht der Malerei (86). Die Struktur der Streifen sei daher auch kein Verweis

17 Siehe dazu zusammenfassend die Ausführungen im Vorwort zur Neuauflage von T. J. Clark: The Painting of Modern Life: Paris in the Art of Manet and His Followers. Überarb. Aufl. Princeton: Princeton UP, 1984. xxii-xxv.

18 Clement Greenberg: „Modernist Painting.“ 1960. The Collected Essays and Criticism. Hg. John O'Brian. 85-93; 86. Ihren Abschluss fand diese Interpretationslinie in Michael Frieds großer Manet-Studie, in der der formalistische Ansatz nur noch gleichberechtigt neben einem phänomenologischen und stilgeschichtlichen steht. Vgl. Manet's Modernism: or, The Face of Painting in the 1860s. Chicago: Chicago UP, 1996. 402-416.

19 Vgl. De Duve: Kant after Duchamp. 203-205; David Hopkins: After Modern Art 1945-2000. Oxford: Oxford UP, 2000. 133-135. 
auf den Bildträger, wie Fried seinerzeit argumentierte, um Stella noch als Grenzfall des Formalismus reklamieren zu können, ${ }^{20}$ sondern „eine die Bildoberfläche überziehende, iterative Struktur, die sich scheinbar selbst hervorgebracht hat" (87). In ihrer Auslegung vergleicht Graw diese scheinbar anonymen und seriellen Bilder mit dem Topos des selbstständigen, subjekthaften Bildes, interpretiert sie mithin als Teil der von ihr konstatierten Dialektik der Lebendigkeit zweiter Ordnung $(90,96)$. Hieran schließt sich eine Besprechung von Ellsworth Kellys und Gerhard Richters Arbeiten an, in der auch diese für die Dialektik der Revitalisierung reklamiert werden sollen. Eine häretische Lesart freilich auch dies, zählen die beiden Künstler für gewöhnlich doch zu einer malerischen Subjektkritik, in der noch die letzte Suggestion von Innerlichkeit gerichtet wird. Was Kelly betrifft, überrascht es dabei jedoch, dass zwar dessen Reliefarbeiten besprochen werden, nicht aber die Monochrombilder, denn mit ihrer Tilgung jeglicher Expressivität sind sie es wohl, die die größte Schwierigkeit für Graws Modell darstellen. ${ }^{21}$ Richters Arbeiten werden in einer präzisen Interpretation - in freundschaftlicher Distanz zu Benjamin H. D. Buchlohs einflussreicher Auslegung ${ }^{22}$ - nicht als reine Subjektkritik interpretiert, sondern als Depotenzierung des Autors, die in umgekehrter Proportion zu einer Belebung des Bildes führe (107).

Das dritte Kapitel setzt die Erzählung der Dialektik von Kritik und Vitalisierung mit der Losung „Malerei gegen Malerei“ fort. Thematisch wird hier die Malereikritik, wie sie in Deutschland von Jörg Immendorff, Sigmar Polke, Martin Kippenberger, Jutta Koether und Albert Oehlen entwickelt wurde. Dabei wird eine Konstellation aufgezeigt zwischen dem Einbruch des Readymade in die Malerei, der „Privilegierung externer Parameter“ und der Aufnahme konzeptueller Elemente. Ergänzt wird diese Diskussion mit einer Studie zur "Persona im Produkt“ bei Kippenberger, vielleicht die beste kunstkritische Passage des Buches, welche auf einen Text zurückgeht, den Graw 2013 für dessen Werkverzeichnis schrieb. Ausgehend von der Diskussion um eine Öffnung der Malerei auf den Lebenskontext hin, analysiert Graw hier auf subtile Weise die Fallstricke einer solchen Konzeption, ohne das Werk je auf Biografisches zu reduzieren. Den Schluss des Kapitels bildet ein Gespräch mit Merlin Carpenter, dem ehemaligen Assistenten Kippenbergers, in welchem dieser mit bemerkenswerter Offenheit von den öko-

20 Michael Friel: Three American Painters: Kenneth Noland, Jules Olitski, Frank Stella. Ausstellungskatalog. Harvard: Harvard UP, 1965.

21 Vgl. etwa die Interpretation bei Branden W. Joseph: Random Order: Robert Rauschenberg and the Neo-Avant-Garde. Cambridge, MA: MIT Press, 2003.97-104.

22 Benjamin H. D. Buchloh: „Readymade, Photography, and Painting in the Painting of Gerhard Richter." 1977. Neo-Avantgarde and Culture Industry: Essays on European and American Art from 1955 to 1975. Cambridge, MA: MIT-Press, 2000. 365-403. 
nomischen und sozialen Bedingungen spricht, unter denen Malerei heute stattfindet. Wer dort spricht, spricht ohne kritizistischen Zynismus, ohne Romantik auch, mit einer Nüchternheit, die in ihrer Drastik zu Ende zu denken wäre: „[D]er traurige Maler, der sein Gemälde in den Mittelpunkt stellt, stellt letztlich seine Klassenstellung in den Mittelpunkt, statt sie zurückzuweisen.“ (202)

Im vierten Kapitel werden schließlich solche Praxen thematisch, die zu diskutieren im Rahmen eines jeden restriktiven Malereibegriffs abwegig scheinen müssten, für Graws Ansatz sich jedoch aufdrängen. Mit einer Geste, die man kaum anders denn als kalkulierte Provokation verstehen kann, wird eingangs der Schutzheilige des Postmedialen, Marcel Broodthaers, als erster Zeuge für diesen radikal entgrenzten Malerbegriff aufgerufen. Graw formuliert ihre Provokation in sechs Thesen, welche zum Teil interessante Überlegungen $\mathrm{zu}$ Anspielungen auf die Malerei bei Broodthaers entwickeln, bei denen zugleich aber unklar bleibt, wie stark Graw ihre These letztlich machen möchte. Exemplarisch zeigt sich dies bei der Diskussion von Broodthaers Poemès industriels, einer viel diskutierten Serie von industriell gepressten, an Werbetafeln erinnernden Schildern. Für sie kann Graw diverse Anspielungen auf die Malerei rekonstruieren, wobei sie jedoch der Frage ausweicht, ob sie diese Arbeiten als Teil eines erweiterten Malereibegriffs lesen möchte, ob es sich um Arbeiten handelt, die sich von jeder Bindung an ein Medium gelöst haben, oder ob es sich bloß um Anspielungen auf Geschichte und Rhetorik der Malerei handelt. Gerade weil in diesem Buch Schluss gemacht werden soll mit einem Begriff der reinen Malerei, wäre es geboten, den Begriff einer unreinen, einer post-medialen Malerei schärfer zu fassen. Zumal, wenn von Randfällen die Rede ist, denn vom phänomenalen Extrem her gewinnt ein Begriff seine Kontur.

Stringenter als die Diskussion von Marcel Broodthaers ist jene von Rachel Harrisons und Isa Genzkens Arbeiten. Der Nachweis einer Anspielung auf die Malerei in diesen, gewöhnlich im Rahmen von Skulptur und Assemblage diskutierten Werken nimmt die Form der These an, dass es vor allem der ,Einsatz der Farbe ist, der diese Assemblagen mit malerischem Potenzial anreichert“ (246). Denn: „Für das latente Anthropomorphe dieser Objekte sorgt, dass ihre Oberflächen entweder, wie bei Harrison, grundsätzlich bemalt oder, wie bei Genzken, mit Farbe angesprüht und mit Folie und Tape beklebt werden.“ (252) In einem zweiten Schritt entwickelt Graw eine recht kritische Auslegung dieser Arbeiten, vor allem von Genzkens Schaufensterpuppen. Die Kombination ihrer deformierten Gestalt mit dem Rekurs auf das vitalistische Potenzial der Farbe generiere eine problematische Identifikationsfläche, denn was diese Arbeiten inszenieren, sei „die Geschichte jenes pathologischen und beschädigten Subjekts [...], die ihren Betrachter/innen wohl vertraut ist“ (263). Es überrascht, dass Graw es für unentschieden hält, ob diesen Arbeiten, in denen das politische und ökonomische Desaster unserer Gegenwart als mimetisches benannt wird, noch ein ausreichend 
kritisches Moment innewohnt, oder ob es sich um bloße Spiegelungen der Warenwelt handelt. Denn „Mimesis ans Verhärtete“ ${ }^{“ 23}$ meint keine Assimilation, sondern Denunziation, meint immanenten Einspruch, nicht Zuspruch zu dem, was ist. ${ }^{24}$

Das fünfte Kapitel stellt eine Auseinandersetzung mit dem Begriff des „network painting“ dar, den David Joselit - ausgehend von Bruno Latours Netzwerktheorie - vor einigen Jahren in einem vielbeachteten Aufsatz geprägt hat. ${ }^{25}$ Mit dieser losen morphologischen Kategorie sollte in erster Linie die Visualisierung derjenigen Netzwerke bezeichnet werden, in denen sich Maler/innen sozial bewegen und welche die Zirkulation ihrer Werke organisieren. Offensichtlich wird bei Joselit aus denselben Gründen auf den Begriff des Netzwerks rekurriert, aus denen Graw für den des Zeichens optiert: um jenen des Mediums zu vermeiden. Gegen Joselits theoriestrategische Alternative wendet Graw ein, dass diese eine geschichtsblinde Perspektive impliziere, mithin verkenne, „dass ein solcher Eintritt des Sozialen oder genauer, des Freundeskreises des Künstlers in die Malerei, kein neues Phänomen ist“ (270), was sie mit Hinweisen auf Francis Picabia und Max Ernst überzeugend darlegen kann. Das letzte Kapitel des Buches kehrt zu materialistischen Überlegungen, genauer: $\mathrm{zu}$ Wertfragen zurück, welche zunächst in zehn Thesen entwickelt und dann in einem sehr lesenswerten Gespräch mit der Theoretikerin Kerstin Stakemeier zur Diskussion gestellt werden.

Wer das Buch nach diesem Kapitel schließt, hat weniger den Eindruck, dass es sich um ein fertiges Projekt handelt, sondern vielmehr um das Dokument eines Denkens, das versucht, eine Momentaufnahme der Malerei zu entwickeln. Eine Beweglichkeit, die vielleicht allein dem Gegenstand angemessen ist. Wichtig ist das Buch daher auch nicht nur, weil es eine anspruchsvolle Theorie der Malerei unter post-medialen Bedingungen formuliert und diese in einer Vielzahl von präzisen Analysen zeitgenössischer Maler/innen erprobt. Wichtig ist es auch dort, wo theoretische Probleme entstehen, Fragen aufgeworfen werden, denn auch darin ist es in seiner Aktualität exemplarisch. Nicht in Erwartung einer innerakademischen Theorie oder eines Versuchs der Kanonisierung der zeitgenössischen Malerei, als Eingriff ins Gemenge sollte man daher Isabelle Graws neues Buch lesen.

23 Theodor W. Adorno: Ästhetische Theorie. Gesammelte Werke. Bd. VII. Hg. Rolf Tiedemann. Frankfurt a.M.: Suhrkamp, 2003. 39.

24 Vgl. dagegen Hal Fosters Interpretation, der ebenfalls den mimetischen Charakter dieser Arbeiten herausarbeitet, dabei aber auf deren kritischer Dimension insistiert: Bad New Days: Art, Criticism, Emergency. London und New York: Verso, 2015. 78-91.

25 David Joselit: „Painting Beside Itself.“ October 130 (2009): 125-134. Der Begriff wurde sodann in einer von Joselit mitkuratierten Ausstellung als zentrale Kategorie für die Beschreibung der Malerei seit 1960 kanonisiert. Vgl. Manuela Ammer, Achim Hochdörfer, David Joselit, Hgg.: Painting 2.0: Expression in the Information Age. Seine Netzwerktheorie hat Joselit von einer Malerei- zu einer globalen Bildtheorie erweitert. Vgl. ders.: After Art. Princeton: Princeton UP, 2013. 